Article

\title{
Radiative Decay Rates for Electric Dipole, Magnetic Dipole and Electric Quadrupole Transitions in Triply Ionized Thulium (Tm IV)
}

\author{
Saturnin Enzonga Yoca ${ }^{1}$ and Pascal Quinet ${ }^{2,3, *}$ \\ 1 Département de Physique, Faculté des Sciences et Techniques, Université Marien Ngouabi, \\ BP 69 Brazzaville, Congo; saturnin.enzongayoca@umng.cg \\ 2 Physique Atomique et Astrophysique, Université de Mons, B-7000 Mons, Belgium \\ 3 IPNAS, Université de Liège, B-4000 Liège, Belgium \\ * Correspondence: Pascal.Quinet@umons.ac.be; Tel.: +32-65-373-629
}

Academic Editor: Robert C. Forrey

Received: 2 August 2017; Accepted: 11 September 2017; Published: 13 September 2017

\begin{abstract}
A new set of radiative decay parameters (oscillator strengths, transition probabilities) for spectral lines in triply ionized thulium (Tm IV) has been obtained within the framework of the pseudo-relativistic Hartree-Fock (HFR) approach. The effects of configuration interaction and core-polarization have been investigated in detail and the quality of the results has been assessed through a comparison between different HFR physical models. The spectroscopic data listed in the present paper cover electric dipole as well as magnetic dipole and electric quadrupole transitions in a wide range of wavelengths from extreme ultraviolet to near infrared.
\end{abstract}

Keywords: atomic structure; oscillator strengths; transition probabilities; Tm IV spectrum

\section{Introduction}

Because of their unusual luminescent properties, triply charged lanthanide ions find many applications in different scientific and technological fields such as the lighting industry, photonics, laser physics and biotechnology, see e.g., [1-6]. If we except $\mathrm{La}^{3+}$, all these ions are characterized by a ground electronic configuration of the type [Xe]4f ${ }^{k}$ with $k$ varying from $1\left(\mathrm{Ce}^{3+}\right)$ to $14\left(\mathrm{Lu}^{3+}\right)$, which is mainly responsible for their interesting photophysical properties such as long-lived luminescence and sharp absorption and emission lines.

Until recently, their radiative properties were essentially investigated from spectroscopy experiments on ions embedded in compounds or crystal lattices. Among those ions, $\mathrm{Tm}^{3+}$ can be considered as being of moderate complexity with $4 \mathrm{f}^{12}$ as ground state configuration. Its emission spectrum has been studied in various compounds such as, for example, in thulium ethylsulphate $[7,8]$, in $\mathrm{CaF}_{2}$ [9], in $\mathrm{CaWO}_{4}$ [10,11], in $\mathrm{YVO}_{4}$ [12], or in $\mathrm{LiYF}_{4}$ [13]. With regard to the free $\mathrm{Tm}^{3+}$ ion, the first spectrum analysis was published just a few years ago by Meftah et al. [14] who gave a selection of some prominent observed lines together with the corresponding transition probabilities estimated using a rather simple theoretical approach.

Let us also mention that triply ionized thulium has been proposed as being suitable for ultraprecise atomic optical clocks with naturally suppressed blackbody-radiation shift of clock transition frequency [15]. This ion has ground and long-living first excited states belonging to the $4 \mathrm{f}^{12}$ configuration, giving rise to electric quadrupole transitions within the optical or infrared wavelength range. Moreover, the width of the first excited state of two-hole $n \mathrm{f}^{12}$ configuration was estimated to be an order of magnitude smaller than that of the two-electron $n \mathrm{f}^{2}$ first excited state with the same transition frequency making ions with $n \mathrm{f}^{12}$ as ground configuration, such as $\operatorname{Tm}^{3+}(n=4)$, interesting 
candidates for providing clock transitions that have quality factors that are many orders of magnitude larger than the ones used in current atomic clocks [16].

In the present work, the radiative parameters corresponding to electric dipole, magnetic dipole and electric quadrupole transitions in Tm IV were computed using the relativistic Hartree-Fock (HFR) method including configuration interaction and core-polarization effects in different levels of approximation. These calculations are an extension of our previous investigations of triply ionized lanthanides La IV [17], Ce IV [18], Pr IV [19], Nd IV [20], and Yb IV [21].

\section{Classified Lines and Term Analysis in Tm IV}

Recently, the emission spectrum of thulium was observed in the spectral region 700-2320 $\AA$ and the first classification of the most important transitions of Tm IV was reported by Meftah et al. [14]. More precisely, using the $10.7 \mathrm{~m}$ normal-incidence vacuum ultraviolet spectrograph installed in Paris-Meudon Observatory, these authors were able to identify more than 760 lines as transitions between 157 levels of $4 f^{11} 5 d$, 33 levels of $4 f^{11} 6 p$, 9 levels of $4 f^{11} 6 s$ and 10 levels of the $4 f^{12}$ ground configuration in Tm IV. A parametric interpretation of the levels was carried out using the Cowan codes [22] by including $4 \mathrm{f}^{12}, 4 \mathrm{f}^{11} 6 \mathrm{p}, 5 \mathrm{p}^{5} 4 \mathrm{f}^{13}$ and $4 \mathrm{f}^{11} 5 \mathrm{~d}, 4 \mathrm{f}^{11} 6 \mathrm{~s}, 5 \mathrm{p}^{5} 4 \mathrm{f}^{12} 5 \mathrm{~d}$ as interacting configurations in the even and odd parities, respectively. In their paper, Meftah et al. [14] mentioned that additional work on Tm IV was foreseen, notably to determine more levels with $J=2$ and $J=1$, as well as the lowest levels of $4 \mathrm{f}^{11} 6 \mathrm{~d}$ and $4 \mathrm{f}^{11} 7 \mathrm{~s}$ but, to our knowledge, no further investigation has been published so far.

\section{Available Transition Rates}

The only set of radiative data for electric dipole transitions available in the literature for Tm IV was published by Meftah et al. [14], who used the semi-empirical relativistic Hartree-Fock approach of Cowan [22] as described in the previous section. They only reported oscillator strengths for 59 transitions involving the $4 \mathrm{f}^{12}{ }^{3} \mathrm{H}_{6}$ ground level and transition probabilities for 46 lines having $4 \mathrm{f}^{11}\left({ }^{4} \mathrm{I}_{15 / 2}\right) 6 \mathrm{p}$ as upper configuration. Moreover, in this study, configuration interaction effects were discussed, in particular with core-excited configurations $5 \mathrm{p}^{5} 4 \mathrm{f}^{13}$ and $5 \mathrm{p}^{5} 4 \mathrm{f}^{12} 5 \mathrm{~d}$ which were shown to significantly reduce the oscillator strengths corresponding to $4 \mathrm{f}^{12}-4 \mathrm{f}^{11} 5 \mathrm{~d}$ transitions.

Using a detailed free ion Hamiltonian, including electrostatic and spin-orbit terms, as well as two-body, three-body, spin-spin, spin-other-orbit, and electrostatically correlated spin-orbit interactions, Dodson and Zia [23] calculated magnetic dipole and electric quadrupole lines and corresponding oscillator strengths within the ground configurations $4 \mathrm{f}^{k}$ throughout the full trivalent lanthanide series, including Tm IV.

\section{Computational Method Used}

The computational procedure used in the present work for modelling the atomic structure and calculating the radiative parameters in Tm IV is the pseudo-relativistic Hartree-Fock (HFR) method, originally introduced by Cowan [22]. In this approach, a set of orbitals are obtained for each electronic configuration included in the model by solving the Hartree-Fock equations for the spherically averaged atom. The equations are the result of the application of the variational principle to the configuration average energies. Two different physical models were employed in the calculations.

In the first model (A), we used the same approach as the one considered previously for the analysis of the isoelectronic ion Er III [24]. More precisely, the configuration sets explicitly retained for the computations were $4 \mathrm{f}^{12}, 4 \mathrm{f}^{11} 6 \mathrm{p}, 4 \mathrm{f}^{11} 7 \mathrm{p}, 4 \mathrm{f}^{11} 5 \mathrm{f}, 4 \mathrm{f}^{11} 6 \mathrm{f}$ for the even parity, and $4 \mathrm{f}^{11} 6 \mathrm{~s}, 4 \mathrm{f}^{11} 7 \mathrm{~s}$, $4 \mathrm{f}^{11} 5 \mathrm{~d}, 4 \mathrm{f}^{11} 6 \mathrm{~d}, 4 \mathrm{f}^{11} 7 \mathrm{~d}$ for the odd parity. The core-valence interactions were included by means of a core-polarization pseudo-potential and a correction to the dipole operator according to the procedure described in many of our previous papers (see e.g., [25-27]). As a reminder, for atomic systems with $n$ valence electrons, this core-polarization potential gives rise to the following one-particle, $V_{P 1}$, and two-particle, $V_{P 2}$, contributions: 


$$
\begin{gathered}
V_{P 1}=-\frac{1}{2} \alpha_{d} \sum_{i=1}^{n} \frac{r_{i}^{2}}{\left(r_{i}^{2}+r_{c}^{2}\right)^{3}} \\
V_{P 2}=-\alpha_{d} \sum_{i>j} \frac{\vec{r}_{i} \cdot \vec{r}_{j}}{\left[\left(r_{i}^{2}+r_{c}^{2}\right)\left(r_{j}^{2}+r_{c}^{2}\right)\right]^{3 / 2}}
\end{gathered}
$$

In addition, the usual dipole radial integral

$$
\int_{0}^{\infty} P_{n l}(r) r P_{n^{\prime} l^{\prime}}(r) d r
$$

has to be replaced by

$$
\int_{0}^{\infty} P_{n l}(r) r\left[1-\frac{\alpha_{d}}{\left(r^{2}+r_{c}^{2}\right)^{3 / 2}}\right] P_{n^{\prime} l^{\prime}}(r) d r-\frac{\alpha_{d}}{r_{c}^{3}} \int_{0}^{r_{c}} P_{n l}(r) r P_{n^{\prime} l^{\prime}}(r) d r
$$

The core-polarization parameters used in this approach were the dipole polarizability of the $\mathrm{Tm}^{4+}$ ionic core as reported by Fraga et al. [28], i.e., $\alpha_{d}=4.32 \mathrm{a}_{0}{ }^{3}$, and the cut-off radius corresponding to the HFR expectation value of $r$ for the outermost core orbital (5p), i.e., $r_{c}=1.37 \mathrm{a}_{0}$, $\mathrm{a}_{0}$ being the Bohr radius. However, as in the case of our previous analysis related to Er III, such core-polarization corrections were not considered in the atomic orbital calculation of the ground configuration $4 \mathrm{f}^{12}$. The $4 \mathrm{f}$ electrons being indeed located deep inside the Xe-like $5 s^{2} 5 p^{6}$ ionic core, the analytical polarization corrections to the dipole operator as introduced in our model [25-27] are no longer valid for transitions involving these electrons. As an alternative, the uncorrected $\langle 4 \mathrm{f}|\mathrm{r}| 5 \mathrm{~d}>$ radial matrix element corresponding to the $4 \mathrm{f}^{12}-4 \mathrm{f}^{11} 5 \mathrm{~d}$ transitions was scaled down by a factor 0.81 , as we proceeded for Er III [24]. As a reminder, this technique was found to give a good agreement between the calculated radiative lifetimes and the accurate experimental values measured for $4 \mathrm{f}^{13} 5 \mathrm{~d}$ levels in $\mathrm{Yb}$ III [29] and $4 \mathrm{f}^{12} 5 \mathrm{~d}$ levels in Tm III [30], which can only decay by $4 \mathrm{f}-5 \mathrm{~d}$ transitions to the $4 \mathrm{f}^{14}$ and $4 \mathrm{f}^{13}$ ground configurations, respectively.

In their observation and interpretation of the Tm IV spectrum, Meftah et al. [14] noticed a significant reduction of the resonance transition probabilities $4 \mathrm{f}-5 \mathrm{~d}$ when the interaction $5 p^{6} 4 f^{11} 5 d-5 p^{5} 4 f^{12} 5 d$ was treated explicitly, this reduction appearing comparable in size to the core-polarization effects included in the close spectrum of $\mathrm{Yb}$ IV [21]. In order to confirm this assertion and verify it for all the transition arrays considered in the present work, i.e., $4 \mathrm{f}^{12}-4 \mathrm{f}^{11} 5 \mathrm{~d}$, $4 \mathrm{f}^{11} 5 \mathrm{~d}-4 \mathrm{f}^{11} 6 \mathrm{p}$ and $4 \mathrm{f}^{11} 6 \mathrm{~s}-4 \mathrm{f}^{11} 6 \mathrm{p}$, we built a second HFR physical model (B) in which the $5 \mathrm{p}^{5} 4 \mathrm{f}^{13}$, $5 p^{5} 4 f^{12} 6 p$ even-parity, and the $5 p^{5} 4 f^{12} 5 d, 5 p^{5} 4 f^{12} 6$ s odd-parity configurations were explicitly added to the set of interacting configurations included in model A. In this case, no additional core-polarization corrections were included.

In both models A and B, the radial energy parameters were adjusted using a well-established semi-empirical least-squares fitting procedure [22] in order to minimize the differences between computed and available experimental energy levels. More precisely, the average energies $\left(E_{a v}\right)$, the Slater integrals for direct $\left(F^{k}\right)$ and exchange $\left(G^{k}\right)$ electrostatic interactions, the spin-orbit integrals $\left(\zeta_{n l}\right)$, and the two-body second-order effective interaction parameters $(\alpha, \beta, \gamma)$ corresponding to the $4 \mathrm{f}^{12}, 4 \mathrm{f}^{11} 6 \mathrm{p}$ even- and the $4 \mathrm{f}^{11} 5 \mathrm{~d}, 4 \mathrm{f}^{11} 6 \mathrm{~s}$ odd-parity configurations, together with the $4 \mathrm{f}^{11} 5 \mathrm{~d}-4 \mathrm{f}^{11} 6 \mathrm{~s}$ configuration interaction integrals $\left(R^{k}\right)$, were adjusted using all the measured level energies listed in [14]. For the configurations that are not experimentally known, Slater integrals were multiplied by a scaling factor (0.85), as suggested by Cowan [22], while the spin-orbit integrals were kept at their $\mathrm{ab}$ initio values. The final results of the fits were found to be almost independent of the model used, the average energy deviations being very close to each other, i.e., $30 \mathrm{~cm}^{-1}$ (model A) and $25 \mathrm{~cm}^{-1}$ (model B) for the even parity, and $117 \mathrm{~cm}^{-1}$ (A) and $105 \mathrm{~cm}^{-1}$ (B) for the odd parity. We note that these values are comparable to the root mean-square deviations of $38 \mathrm{~cm}^{-1}$ (even levels) and $54 \mathrm{~cm}^{-1}$ 
(odd levels) obtained by Meftah et al. [14] using a more restricted set of interacting configurations than those considered in our calculations. The differences in standard deviations, in particular in the odd parity, can be explained by the consideration of more extended multiconfiguration expansions in our models that introduce more uncertainties in our fits due to the unknown positions of additional configurations. However, the use of these more elaborate models are necessary to obtain reliable radiative transition rates, which was not the main goal of the work published by Meftah et al. [14], who were rather focused on the interpretation of the Tm IV energy level structure.

\section{Electric Dipole Transitions}

The oscillator strengths ( $\log g f)$ computed in the present work using our two physical models, A and B, are compared to those reported by Meftah et al. [14] in Tables 1 and 2 for transitions involving the ground level $4 \mathrm{f}^{12}{ }^{3} \mathrm{H}_{6}$ and the $4 \mathrm{f}^{11}\left({ }^{4} \mathrm{I}_{15 / 2}\right) 6 \mathrm{p}$ subconfiguration, respectively. In their investigation, the latter authors evaluated the importance of opening the $5 \mathrm{p}^{6}$ subshell on the oscillator strengths by comparing HFR calculations with and without $5 \mathrm{p}^{5} 4 \mathrm{f}^{13}$ and $5 \mathrm{p}^{5} 4 \mathrm{f}^{12} 5 \mathrm{~d}$ configurations. In fact, when adding these two configurations to a rather simple model including $4 \mathrm{f}^{12}, 4 \mathrm{f}^{11} 6 \mathrm{p}$ and $4 \mathrm{f}^{11} 5 \mathrm{~d}, 4 \mathrm{f}^{11} 6 \mathrm{~s}$ in the even and odd parities, respectively, they found that the $\log g f$-values corresponding to the $4 f^{12}-4 f^{11} 5 d$ resonance transitions were reduced by an average value of 0.18 while the $4 f^{11} 6 s-4 f^{11} 6 p$ and $4 f^{11} 5 d-4 f^{11} 6 p$ transition rates were almost unaffected. When looking at Table 1 , we note that, for $4 \mathrm{f}^{12}-4 \mathrm{f}^{11} 5 \mathrm{~d}$ resonance transitions, the results obtained with our model A are in very good agreement with the oscillator strengths computed by Meftah et al. [14]; using their model including the $5 p^{5} 4 \mathrm{f}^{13}$ and $5 p^{5} 4 f^{12} 5 d$ configurations, the mean deviation in $\log g f$ being found to be equal to 0.02 . In other words, for the $4 \mathrm{f}-5 \mathrm{~d}$ transitions, the core-polarization corrections considered in our model A lead to results comparable to those obtained by Meftah et al. [14] when opening the $5 p^{6}$ subshell. However, this is no more the case when looking at the $5 \mathrm{~d}-6 \mathrm{p}$ and $6 \mathrm{~s}-6 \mathrm{p}$ transitions listed in Table 2. Indeed, for these transitions, the oscillator strengths calculated with our Model A appear systematically smaller than the values obtained by Meftah et al. [14] or by using our Model B, the mean ratios being found to be equal to $g f(\mathrm{~A}) / g f[14]=0.77 \pm 0.07$ and $g f(\mathrm{~A}) / g f(\mathrm{~B})=0.79 \pm 0.03$. This implies that the core-excited configurations considered in these two latter approaches (by promoting one electron from the $5 p$ core orbital only) are not sufficient to entirely take the core-valence electron correlations into account while the core-polarization corrections used in our calculation A are expected to model not only single excitations from $5 p$ but also from other core subshells such as $5 \mathrm{~s}$ and $4 \mathrm{f}$, for example.

As there are no experimental radiative data available in the literature for $\mathrm{Tm} I \mathrm{~V}$, a possible way of assessing the reliability of the results obtained in the present work lies in isoelectronic comparisons, particularly with the study performed in Er III a few years ago [24]. In this work, radiative lifetimes of seven excited states belonging to the $4 \mathrm{f}^{11} 6 \mathrm{p}$ configuration, in the energy range from 55,547 to $81,838 \mathrm{~cm}^{-1}$, have been measured using the time-resolved laser-induced fluorescence technique. The comparison of these accurate experimental data with core-polarization corrected HFR calculations, using the same physical model as the one considered in model A of the present work, has shown that the computed values were in excellent agreement (within 8\%) with the measurements. An identical agreement (within 10\%) was also found when comparing a similar theoretical model with experimental radiative lifetimes for $4 \mathrm{f}^{12} 6 \mathrm{p}$ levels between 76,721 and $82,573 \mathrm{~cm}^{-1}$ in Tm III [30]. An accuracy of the same order of magnitude can therefore also be expected for the radiative decay rates computed in the present work for transitions depopulating the $4 \mathrm{f}^{11} 6 \mathrm{p}$ levels in $\mathrm{Tm} \mathrm{IV}$, at least for the most intense lines. 
Table 1. Calculated oscillator strengths $(\log g f)$ for transitions involving the lower $4 \mathrm{f}^{12}{ }^{3} \mathrm{H}_{6}$ ground level to upper $4 \mathrm{f}^{11} 5 \mathrm{~d}$ levels in Tm IV.

\begin{tabular}{|c|c|c|c|c|c|c|}
\hline \multirow{2}{*}{$\lambda(\AA)^{1}$} & \multicolumn{2}{|c|}{ Transition } & \multicolumn{2}{|c|}{$\log g f$ (This Work) } & \multicolumn{2}{|c|}{$\log g f[14]$} \\
\hline & Lower Level ${ }^{2}$ & Upper Level $^{2}$ & $A^{3}$ & $\mathrm{~B}^{4}$ & no $\mathrm{CI}^{5}$ & $\mathrm{CI}^{6}$ \\
\hline 748.928 & $0.00(6)$ & $133524.48(5)$ & -2.21 & -2.35 & -1.83 & -2.00 \\
\hline 781.617 & $0.00(6)$ & $127939.33(6)$ & -2.05 & -2.04 & -1.89 & -2.00 \\
\hline 787.460 & $0.00(6)$ & $126990.55(5)$ & -1.12 & -1.43 & -1.01 & -1.27 \\
\hline 792.742 & $0.00(6)$ & $126144.12(6)$ & -1.83 & -1.86 & -1.58 & -1.73 \\
\hline 793.374 & $0.00(6)$ & $126043.76(7)$ & -1.95 & -1.98 & -1.96 & -2.02 \\
\hline 802.337 & $0.00(6)$ & $124636.37(5)$ & -1.43 & -2.41 & -1.38 & -1.83 \\
\hline 804.050 & $0.00(6)$ & $124370.09(6)$ & -1.25 & -1.33 & -1.18 & -1.32 \\
\hline 811.339 & $0.00(6)$ & $123253.77(6)$ & -2.26 & -2.56 & -1.99 & -2.23 \\
\hline 812.435 & $0.00(6)$ & $123086.74(5)$ & -0.61 & -0.79 & -0.48 & -0.69 \\
\hline 827.296 & $0.00(6)$ & $120875.97(5)$ & -0.69 & -0.85 & -0.51 & -0.73 \\
\hline 829.041 & $0.00(6)$ & $120621.39(6)$ & -1.81 & -2.03 & -1.73 & -1.92 \\
\hline 831.785 & $0.00(6)$ & $120223.30(7)$ & -0.96 & -1.28 & -0.88 & -1.14 \\
\hline 839.626 & $0.00(6)$ & $119100.52(7)$ & -0.91 & -1.02 & -0.72 & -0.92 \\
\hline 839.927 & $0.00(6)$ & $119057.80(5)$ & -2.88 & -3.08 & -2.34 & -2.54 \\
\hline 850.291 & $0.00(6)$ & $117607.00(6)$ & -1.77 & -1.88 & -1.65 & -1.81 \\
\hline 850.838 & $0.00(6)$ & $117531.05(7)$ & -0.21 & -0.38 & -0.08 & -0.29 \\
\hline 863.720 & $0.00(6)$ & $115778.36(5)$ & -2.00 & -2.19 & -1.72 & -1.95 \\
\hline 875.898 & $0.00(6)$ & $114168.84(5)$ & -2.33 & -2.65 & -2.79 & -2.92 \\
\hline 888.951 & $0.00(6)$ & $112491.99(5)$ & -0.61 & -0.86 & -0.38 & -0.60 \\
\hline 890.547 & $0.00(6)$ & $112290.52(7)$ & -1.18 & -1.32 & -1.05 & -1.24 \\
\hline 893.806 & $0.00(6)$ & $111881.49(5)$ & -0.91 & -0.91 & -1.22 & -1.31 \\
\hline 897.819 & $0.00(6)$ & $111381.45(6)$ & -1.02 & -1.23 & -0.81 & -1.14 \\
\hline 898.984 & $0.00(6)$ & $111236.63(5)$ & -2.11 & -2.43 & -1.29 & -1.28 \\
\hline 903.148 & $0.00(6)$ & $110723.68(5)$ & -0.87 & -1.00 & -0.74 & -0.92 \\
\hline 908.328 & $0.00(6)$ & $110092.40(6)$ & -1.69 & -1.77 & -1.31 & -1.53 \\
\hline 913.550 & $0.00(6)$ & $109463.34(5)$ & -1.26 & -1.33 & -1.26 & -1.41 \\
\hline 916.354 & $0.00(6)$ & $109128.14(6)$ & -1.37 & -1.44 & -1.23 & -1.39 \\
\hline 926.904 & $0.00(6)$ & $107885.74(7)$ & -1.00 & -1.10 & -0.87 & -1.08 \\
\hline 929.338 & $0.00(6)$ & $107603.36(6)$ & -1.47 & -1.63 & -0.31 & -0.91 \\
\hline 932.017 & $0.00(6)$ & $107294.13(6)$ & -0.34 & -0.45 & -0.94 & -0.55 \\
\hline 935.493 & $0.00(6)$ & $106895.45(7)$ & -1.30 & -1.46 & -1.40 & -1.51 \\
\hline 942.489 & $0.00(6)$ & $106101.98(6)$ & -1.20 & -1.24 & -1.26 & -1.39 \\
\hline 944.471 & $0.00(6)$ & $105879.41(6)$ & -0.87 & -1.03 & -0.68 & -0.88 \\
\hline 950.964 & $0.00(6)$ & $105156.00(5)$ & -0.98 & -1.09 & -0.91 & -1.09 \\
\hline 963.402 & $0.00(6)$ & $103799.19(6)$ & -0.87 & -1.02 & -0.84 & -1.03 \\
\hline 980.201 & $0.00(6)$ & $102020.08(6)$ & -1.82 & -1.95 & -1.61 & -1.81 \\
\hline 981.108 & $0.00(6)$ & $101925.68(5)$ & -1.32 & -1.46 & -1.46 & -1.70 \\
\hline 999.724 & $0.00(6)$ & $100027.46(5)$ & -1.58 & -1.66 & -1.48 & -1.65 \\
\hline 1024.207 & $0.00(6)$ & $97637.17(5)$ & -1.96 & -2.34 & -1.90 & -2.04 \\
\hline 1031.126 & $0.00(6)$ & $96982.12(6)$ & -2.14 & -2.09 & -2.04 & -2.16 \\
\hline 1045.098 & $0.00(6)$ & $95685.30(6)$ & -2.04 & -2.20 & -2.06 & -2.24 \\
\hline 1048.446 & $0.00(6)$ & $95379.52(5)$ & -1.66 & -1.76 & -1.48 & -1.65 \\
\hline 1054.249 & $0.00(6)$ & $94854.50(5)$ & -1.40 & -1.52 & -1.34 & -1.54 \\
\hline 1062.470 & $0.00(6)$ & $94119.93(6)$ & -0.90 & -1.01 & -0.87 & -1.05 \\
\hline 1080.102 & $0.00(6)$ & $92583.34(6)$ & -1.08 & -1.10 & -0.88 & -1.03 \\
\hline 1098.150 & $0.00(6)$ & 91061.55 (7) & -1.50 & -1.66 & -1.41 & -1.63 \\
\hline 1133.437 & $0.00(6)$ & $88226.85(6)$ & -1.92 & -1.92 & -1.60 & -1.75 \\
\hline 1138.014 & $0.00(6)$ & $87872.31(7)$ & -1.61 & -1.72 & -1.67 & -1.84 \\
\hline 1148.226 & $0.00(6)$ & $87090.72(5)$ & -2.94 & -2.91 & -2.47 & -2.67 \\
\hline 1153.166 & $0.00(6)$ & $86717.61(6)$ & -0.81 & -0.88 & -0.80 & -0.95 \\
\hline 1160.827 & $0.00(6)$ & $86145.56(7)$ & -1.26 & -1.37 & -1.30 & -1.47 \\
\hline 1169.015 & $0.00(6)$ & 85542.07 (5) & -1.45 & -1.57 & -1.46 & -1.64 \\
\hline 1183.627 & $0.00(6)$ & $84485.81(6)$ & -1.02 & -1.10 & -0.86 & -1.04 \\
\hline 1197.175 & $0.00(6)$ & $83530.02(7)$ & -1.15 & -1.21 & -1.04 & -1.21 \\
\hline 1200.580 & $0.00(6)$ & 83293.13 (5) & -1.57 & -1.66 & -1.42 & -1.60 \\
\hline 1245.880 & $0.00(6)$ & $80264.65(7)$ & -1.26 & -1.34 & -1.08 & -1.26 \\
\hline 1262.212 & $0.00(6)$ & 79225.87 (6) & -1.56 & -1.64 & -1.42 & -1.59 \\
\hline 1275.288 & $0.00(6)$ & $78413.63(5)$ & -2.50 & -2.60 & -2.33 & -2.53 \\
\hline
\end{tabular}

Notes: ${ }^{1}$ Experimental wavelengths from [14]; ${ }^{2}$ Experimental energy levels in $\mathrm{cm}^{-1}$ from [14]. J-values are given between parentheses; ${ }^{3}$ Model A used in the present work (see text); ${ }^{4}$ Model B used in the present work (see text); ${ }^{5}$ Calculations reported in [14] not including $5 \mathrm{p}^{5} 4 \mathrm{f}^{13}$ and $5 \mathrm{p}^{5} 4 \mathrm{f}^{12} 5 \mathrm{~d} ;{ }^{6}$ Calculations reported in [14] including $5 \mathrm{p}^{5} 4 \mathrm{f}^{13}$ and $5 p^{5} 4 f^{12} 5 d$. 
Table 2. Calculated oscillator strengths (log $g f$ ) for transitions involving lower $4 \mathrm{f}^{11} 5 \mathrm{~d}$ and $4 \mathrm{f}^{11} 6$ s levels to the upper $4 \mathrm{f}^{11}\left({ }^{4} \mathrm{I}_{15 / 2}\right) 6 \mathrm{p}$ subconfiguration in Tm IV.

\begin{tabular}{|c|c|c|c|c|c|c|}
\hline \multirow{2}{*}{$\lambda(\AA)^{1}$} & \multicolumn{2}{|c|}{ Transition } & \multicolumn{2}{|c|}{$\log g f$ (This Work) } & \multicolumn{2}{|c|}{$\log g f[14]$} \\
\hline & Lower Level $^{2}$ & Upper Level $^{2}$ & $\mathrm{~A}^{3}$ & $\mathrm{~B}^{4}$ & no $\mathrm{CI}^{5}$ & $\mathrm{CI}^{6}$ \\
\hline 1212.409 & $72011.02(6)$ & $154491.66(6)$ & -1.30 & -1.22 & -1.20 & -1.21 \\
\hline 1212.792 & $72011.02(6)$ & $154466.21(7)$ & -0.67 & -0.57 & -0.56 & -0.55 \\
\hline 1226.090 & $72931.67(7)$ & $154491.66(6)$ & -1.47 & -1.37 & -1.36 & -1.36 \\
\hline 1226.478 & $72931.67(7)$ & $154466.21(7)$ & -1.16 & -1.07 & -1.05 & -1.05 \\
\hline 1234.245 & $72931.67(7)$ & $153952.89(8)$ & -0.29 & -0.19 & -0.16 & -0.16 \\
\hline 1258.711 & $74506.41(9)$ & $153952.89(8)$ & -1.07 & -1.00 & -0.87 & -0.88 \\
\hline 1267.731 & $75585.02(8)$ & $154466.21(7)$ & -0.97 & -0.87 & -0.79 & -0.81 \\
\hline 1270.461 & $74506.41(9)$ & $153217.84(9)$ & -0.30 & -0.20 & -0.23 & -0.22 \\
\hline 1276.030 & $75585.02(8)$ & $153952.89(8)$ & -0.65 & -0.55 & -0.58 & -0.58 \\
\hline 1288.111 & $75585.02(8)$ & $153217.84(9)$ & -0.64 & -0.54 & -0.56 & -0.55 \\
\hline 1314.439 & $78413.63(5)$ & $154491.66(6)$ & -0.58 & -0.51 & -0.51 & -0.50 \\
\hline 1328.460 & $78677.88(9)$ & $153952.89(8)$ & 0.35 & 0.44 & 0.45 & 0.45 \\
\hline 1329.077 & $79225.87(6)$ & $154466.21(7)$ & -0.50 & -0.42 & -0.39 & -0.39 \\
\hline 1341.565 & $78677.88(9)$ & $153217.84(9)$ & -0.05 & 0.05 & 0.09 & 0.08 \\
\hline 1345.118 & $80122.71(8)$ & $154466.21(7)$ & -0.20 & -0.09 & -0.07 & -0.07 \\
\hline 1347.219 & $80264.65(7)$ & $154491.66(6)$ & -0.79 & -0.69 & -0.63 & -0.63 \\
\hline 1347.679 & $80264.65(7)$ & $154466.21(7)$ & -0.17 & -0.07 & -0.02 & -0.02 \\
\hline 1354.448 & $80122.71(8)$ & $153952.89(8)$ & 0.09 & 0.19 & 0.22 & 0.21 \\
\hline 1357.073 & 80264.65 (7) & $153952.89(8)$ & -0.77 & -0.67 & -0.63 & -0.63 \\
\hline 1368.085 & $80122.71(8)$ & $153217.84(9)$ & -0.96 & -0.86 & -0.81 & -0.82 \\
\hline 1370.232 & $72011.02(6)$ & $144991.40(7)$ & 0.24 & 0.33 & 0.36 & 0.36 \\
\hline 1376.791 & 72931.67 (7) & $145564.25(8)$ & -0.31 & -0.21 & -0.20 & -0.20 \\
\hline 1384.904 & $82258.89(8)$ & $154466.21(7)$ & -0.13 & -0.03 & -0.06 & -0.06 \\
\hline 1387.735 & 72931.67 (7) & $144991.40(7)$ & 0.08 & 0.18 & 0.20 & 0.20 \\
\hline 1394.817 & $82258.89(8)$ & $153952.89(8)$ & -0.82 & -0.76 & -0.89 & -0.86 \\
\hline 1404.524 & 83293.13 (5) & $154491.66(6)$ & -0.01 & 0.09 & 0.12 & 0.11 \\
\hline 1407.305 & 74506.41 (9) & $145564.25(8)$ & 0.38 & 0.48 & 0.50 & 0.50 \\
\hline 1409.212 & $83530.02(7)$ & $154491.66(6)$ & -0.40 & -0.29 & -0.30 & -0.30 \\
\hline 1409.721 & $83530.02(7)$ & $154466.21(7)$ & -0.27 & -0.16 & -0.19 & -0.19 \\
\hline 1420.008 & $83530.02(7)$ & $153952.89(8)$ & -1.26 & -1.15 & -1.22 & -1.21 \\
\hline 1428.465 & $84485.81(6)$ & $154491.66(6)$ & -0.27 & -0.17 & -0.13 & -0.14 \\
\hline 1429.001 & $75585.02(8)$ & $145564.25(8)$ & 0.13 & 0.23 & 0.23 & 0.23 \\
\hline 1440.801 & $75585.02(8)$ & $144991.40(7)$ & -0.15 & -0.05 & -0.04 & -0.04 \\
\hline 1531.399 & 80264.65 (7) & $145564.25(8)$ & -1.17 & -1.07 & -1.06 & -1.07 \\
\hline 1544.960 & 80264.65 (7) & $144991.40(7)$ & -1.00 & -0.91 & -0.93 & -0.93 \\
\hline 1615.952 & $92583.34(6)$ & $154466.21(7)$ & -1.50 & -1.36 & -1.37 & -1.36 \\
\hline 1802.012 & $98972.81(8)$ & $154466.21(7)$ & 0.01 & 0.15 & 0.13 & 0.13 \\
\hline 1818.839 & $98972.81(8)$ & $153952.89(8)$ & 0.55 & 0.69 & 0.71 & 0.71 \\
\hline 1840.039 & $100145.04(7)$ & $154491.66(6)$ & 0.57 & 0.69 & 0.68 & 0.68 \\
\hline 1840.910 & $100145.04(7)$ & $154466.21(7)$ & 0.59 & 0.71 & 0.71 & 0.71 \\
\hline 1843.486 & $98972.81(8)$ & $153217.84(9)$ & 0.83 & 0.97 & 0.97 & 0.97 \\
\hline 1858.450 & $100145.04(7)$ & $153952.89(8)$ & 0.37 & 0.49 & 0.48 & 0.48 \\
\hline 2145.639 & $98972.81(8)$ & $145564.25(8)$ & 0.28 & 0.42 & 0.42 & 0.42 \\
\hline 2172.361 & $98972.81(8)$ & $144991.40(7)$ & 0.55 & 0.69 & 0.69 & 0.69 \\
\hline 2201.026 & $100145.04(7)$ & $145564.25(8)$ & 0.50 & 0.62 & 0.63 & 0.63 \\
\hline 2229.134 & 100145.04 (7) & $144991.40(7)$ & -0.03 & 0.10 & 0.09 & 0.09 \\
\hline
\end{tabular}

Notes: ${ }^{1}$ Experimental wavelengths from [14]; ${ }^{2}$ Experimental energy levels in $\mathrm{cm}^{-1}$ from [14]. J-values are given between parentheses; ${ }^{3}$ Model A used in the present work (see text); ${ }^{4}$ Model B used in the present work (see text); ${ }^{5}$ Calculations reported in [14] not including $5 \mathrm{p}^{5} 4 \mathrm{f}^{13}$ and $5 \mathrm{p}^{5} 4 \mathrm{f}^{12} 5 \mathrm{~d} ;{ }^{6}$ Calculations reported in [14] including $5 \mathrm{p}^{5} 4 \mathrm{f}^{13}$ and $5 p^{5} 4 f^{12} 5 d$.

For all of these reasons, the model A used in the present work is expected to be the most reliable and, as a consequence, oscillator strengths $(\log g f)$ and transition probabilities $(g A)$ computed with this model are reported as supplementary material in Table S1. In the latter, all Tm IV lines involving experimentally known levels for which the $\log g f$ values are greater than -4 are listed. This corresponds to 2913 spectral lines covering a wide wavelength range, from extreme ultraviolet to visible, or, more precisely, from 719 to $5325 \AA$. 


\section{Magnetic Dipole and Electric Quadrupole Transitions}

It is well known that triply charged lanthanide ions exhibit very characteristic emission lines in the visible and near infrared regions due to $4 \mathrm{f} \rightarrow 4 \mathrm{f}$ transitions. These transitions, forbidden by the electric dipole selection rules, are characterized by long lifetimes which facilitate 'time gated' emission experiments leading to significant improvement of signal-to-noise ratios in comparison to more traditional steady-state measurements. Therefore, radiative decay rates of such forbidden lines within the $4 \mathrm{f}^{12}$ ground configuration of Tm IV were also computed in the present work. Transition probabilities obtained using our physical model A for the most intense magnetic dipole (M1) and electric quadrupole (E2) lines are reported in Table 3. They are compared with the results recently published by Dodson and Zia [23] who calculated radiative transition probabilities for some M1 and E2 Tm IV lines using a detailed free ion Hamiltonian including electrostatic and spin-orbit terms as well as two-body, three-body, spin-spin, spin-other-orbit and electrostatically correlated spin-orbit interactions. When looking at Table 3, we can note that their results are generally in good agreement (within a few percent) with our transition probabilities, if we except some E2 transitions located at 3038.774, 3847.249, 4264.491, 4437.432, 4721.408, 5759.349, 6401.911 and $7967.695 \AA$ for which larger discrepancies are observed. However, we note that, for these particular transitions, the cancellation factor as defined by Cowan [22] is very small $(<0.05)$, indicating that the corresponding decay rates might be affected by larger uncertainties in our calculations. For all of the other forbidden lines reported in Table 3 , an accuracy of about $10 \%$ can reasonably be estimated for our computed $g A$-values in view of their excellent agreement with the results obtained using a completely independent theoretical method [23].

Table 3. Transition probabilities for forbidden lines within the $4 \mathrm{f}^{12}$ ground-state configuration of Tm IV. Only transitions with $g A$-values greater than $0.01 \mathrm{~s}^{-1}$ (according to our model A) are listed.

\begin{tabular}{|c|c|c|c|c|c|}
\hline \multirow{2}{*}{$\lambda(\AA)^{1}$} & \multicolumn{2}{|c|}{ Transition } & \multirow[b]{2}{*}{ Type $^{3}$} & \multicolumn{2}{|c|}{$g A\left(\mathrm{~s}^{-1}\right)$} \\
\hline & Lower Level $^{2}$ & Upper Level $^{2}$ & & This Work $^{4}$ & Other ${ }^{5}$ \\
\hline 2829.678 & $0(6)$ & $35329(6)$ & M1 & $1.52 \mathrm{E}+02$ & \\
\hline 3038.774 & $5634(4)$ & $38532(2)$ & E2 & $7.51 \mathrm{E}-01$ & $7.50 \mathrm{E}-02$ \\
\hline 3366.570 & $5634(4)$ & $35329(6)$ & E2 & $1.83 \mathrm{E}-01$ & $2.00 \mathrm{E}-01$ \\
\hline 3687.275 & $8217(5)$ & $35329(6)$ & M1 & $4.94 \mathrm{E}+01$ & $4.56 \mathrm{E}+01$ \\
\hline 3847.249 & $12547(4)$ & $38532(2)$ & E2 & $4.47 \mathrm{E}-01$ & $5.95 \mathrm{E}-02$ \\
\hline 4144.416 & $14410(3)$ & $38532(2)$ & $\mathrm{M} 1+\mathrm{E} 2$ & $9.10 \mathrm{E}+01$ & $1.15 \mathrm{E}+02$ \\
\hline 4264.491 & $15090(2)$ & $38532(2)$ & $\mathrm{M} 1+\mathrm{E} 2$ & $1.32 \mathrm{E}-01$ & $1.79 \mathrm{E}+00$ \\
\hline 4388.182 & 12547 (4) & $35329(6)$ & E2 & $5.05 \mathrm{E}-02$ & $5.99 \mathrm{E}-02$ \\
\hline 4437.432 & $5634(4)$ & $28163(2)$ & E2 & $4.24 \mathrm{E}-01$ & $7.50 \mathrm{E}-02$ \\
\hline 4721.408 & $0(6)$ & $21174(4)$ & E2 & $2.94 \mathrm{E}-02$ & $6.93 \mathrm{E}-03$ \\
\hline 5759.349 & $21174(4)$ & $38532(2)$ & E2 & $1.22 \mathrm{E}-01$ & $1.81 \mathrm{E}-02$ \\
\hline 6401.911 & $12547(4)$ & $28163(2)$ & E2 & $1.45 \mathrm{E}-02$ & $2.40 \mathrm{E}-03$ \\
\hline 6433.154 & $5634(4)$ & $21174(4)$ & M1 & $1.59 \mathrm{E}+01$ & $1.72 \mathrm{E}+01$ \\
\hline 7062.639 & $21174(4)$ & $35329(6)$ & E2 & $1.55 \mathrm{E}-02$ & $1.46 \mathrm{E}-02$ \\
\hline 7269.222 & $14410(3)$ & $28163(2)$ & M1 & $8.99 \mathrm{E}+01$ & $7.00 \mathrm{E}+01$ \\
\hline 7646.868 & $15090(2)$ & $28163(2)$ & M1 & $5.26 \mathrm{E}+01$ & $4.65 \mathrm{E}+01$ \\
\hline 7715.433 & 8217 (5) & $21174(4)$ & M1 & $2.08 \mathrm{E}+02$ & $2.03 \mathrm{E}+02$ \\
\hline 7967.695 & $0(6)$ & $12547(4)$ & E2 & $1.05 \mathrm{E}-02$ & $1.73 \mathrm{E}-04$ \\
\hline 9641.292 & $28163(2)$ & $38532(2)$ & M1 & $6.33 \mathrm{E}+01$ & $6.50 \mathrm{E}+01$ \\
\hline 11391.088 & $5634(4)$ & $14410(3)$ & M1 & $7.68 \mathrm{E}+01$ & $7.63 \mathrm{E}+01$ \\
\hline 11588.383 & $12547(4)$ & $21174(4)$ & M1 & $5.26 \mathrm{E}+01$ & $5.04 \mathrm{E}+01$ \\
\hline 12166.962 & $0(6)$ & 8217 (5) & M1 & $1.61 \mathrm{E}+02$ & $1.60 \mathrm{E}+02$ \\
\hline 14461.107 & $5634(4)$ & $12547(4)$ & M1 & $3.56 \mathrm{E}+01$ & $3.56 \mathrm{E}+01$ \\
\hline 14780.571 & $14410(3)$ & $21174(4)$ & M1 & $5.64 \mathrm{E}+00$ & $5.84 \mathrm{E}+00$ \\
\hline 23085.722 & 8217 (5) & $12547(4)$ & M1 & $1.43 \mathrm{E}+01$ & \\
\hline 38708.464 & $5634(4)$ & 8217 (5) & M1 & $4.09 \mathrm{E}-01$ & \\
\hline 53657.049 & $12547(4)$ & $14410(3)$ & M1 & $3.42 \mathrm{E}-01$ & \\
\hline
\end{tabular}

Notes: ${ }^{1}$ Wavelengths in air deduced from the experimental energy levels taken from [14]; ${ }^{2}$ Experimental energy levels in $\mathrm{cm}^{-1}$ from [14]. J-values are given between parentheses; ${ }^{3} \mathrm{M} 1$ : magnetic dipole; E2: electric quadrupole;

${ }^{4}$ Model A used in the present work (see text); ${ }^{5}$ Dodson and Zia [23]. 


\section{Conclusions}

Oscillator strengths and transition probabilities for allowed and forbidden lines in triply ionized thulium have been obtained using a pseudo-relativistic Hartree-Fock model including core-polarization effects. Due to the lack of experimental data in this ion, the reliability of the new radiative parameters for electric dipole lines has been discussed and assessed through detailed comparisons with different calculations, explicitly including some configurations with an open $5 p^{6}$ subshell, on the one hand, and through the excellent agreement observed between similar calculations and accurate laser lifetime measurements performed in the isoelectronic Er III and isonuclear Tm III ions, on the other hand. The new data concern about $3000 \mathrm{Tm}$ IV spectral lines involving all the experimentally known energy levels in the $4 \mathrm{f}^{12}, 4 \mathrm{f}^{11} 5 \mathrm{~d}, 4 \mathrm{f}^{11} 6 \mathrm{~s}$ and $4 \mathrm{f}^{11} 6 \mathrm{p}$ configurations. In the case of forbidden lines within the $4 \mathrm{f}^{12}$ ground configuration, our results have been found to be in good agreement with theoretical data recently published, if we except a few electric quadrupole transitions.

Supplementary Materials: The following table is available online at www.mdpi.com/2218-2004/5/3/28/s1, Table S1: Calculated oscillator strengths $(\log g f)$ and transition probabilities $(g A)$ for Tm IV spectral lines. Wavelengths in vacuum (air) below (above) $2000 \AA$ are deduced from the experimental energy levels reported in [14]. These levels are given with their rounded energies, parities and J-values in the present table (see [14] for more complete spectroscopic designations and accurate energies).

Acknowledgments: Saturnin Enzonga Yoca was financially supported by a grant from the ANGLE Project (AN13AC2971) and from the Marien Ngouabi University (Congo). Pascal Quinet is Research Director of the Belgian National Fund for Scientific Research F.R.S.-FNRS. Financial support from this organization is acknowledged.

Author Contributions: Both authors were equally involved in the calculations reported in the present paper as well as in the the writing of the manuscript.

Conflicts of Interest: The authors declare no conflict of interest.

\section{References}

1. Hemmilä, I. Luminescent lanthanide chelates-a way to diagnostic methods. J. Alloys Compd. 1995, 225, 480-485. [CrossRef]

2. Wybourne, B.G. The fascination of the rare earths-Then, now and in the future. J. Alloys Compd. 2004, 380, 96-100. [CrossRef]

3. Hasegawa, Y.; Wada, Y.; Yanagida, S. Strategies for the design of luminescent lanthanide(III) complexes and their photonic applications. J. Photochem. Photobiol. C 2004, 5, 183-202. [CrossRef]

4. Walsh, B.M.; Barnes, N.P.; Petros, M.; Yu, J.; Singh, U.N. Spectroscopy and modeling of solid state lanthanide lasers: Application to trivalent $\mathrm{Tm}^{3+}$ and $\mathrm{Ho}^{3+}$ in $\mathrm{YLiF}_{4}$ and LuLiF 4 . J. Appl. Phys. 2004, 95, 3255-3271. [CrossRef]

5. Dossing, A. Luminescence from lanthanide(3+) ions in solution. Eur. J. Inorg. Chem. 2005, 2005, 1425-1434. [CrossRef]

6. Suyver, J.F.; Aebischer, A.; Biner, D.; Gerner, P.; Grimm, J.; Heer, S.; Krämer, K.W.; Reinhard, C.; Güdel, H.U. Novel materials doped with trivalent lanthanides and transition metal ions showing near-infrared to visible photon upconversion. Opt. Mater. 2005, 27, 1111-1130. [CrossRef]

7. Wong, E.Y.; Richman, I. Analysis of the absorption spectrum and Zeeman effect of thulium ethylsulphate. J. Chem. Phys. 1961, 34, 1182-1185. [CrossRef]

8. Krupke, W.F.; Gruber, J.B. Optical-absorption intensities of rare-earth ions in crystals: The absorption spectrum of thulium ethyl sulfate. Phys. Rev. A 1965, 139, A2008. [CrossRef]

9. Muto, K. Absorption spectra and Zeeman effects of $\mathrm{Tm}^{3+}$ in $\mathrm{CaF}_{2}$. J. Phys. Chem. Solids 1973, 34, $2029-2035$. [CrossRef]

10. Wotman, D.E.; Morrison, C.A.; Leavitt, R.P. Analysis of the Ground Configuration of $\mathrm{Tm}^{3+}$ in $\mathrm{CaWO}_{4}$; Harry Diamond Laboratories Report HDL-TR-1663: Washington, DC, USA, 1974.

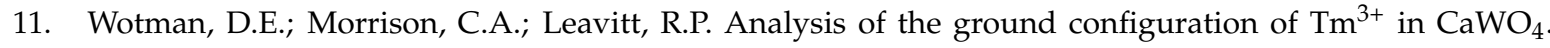
Phys. Rev. B 1975, 12, 4780. [CrossRef]

12. Wotman, D.E.; Leavitt, R.P.; Morrison, C.A. Analysis of the ground configuration of $\mathrm{Tm}^{3+}$ in $\mathrm{YVO}_{4}$. J. Phys. Chem. Solids 1974, 35, 591-593. [CrossRef] 
13. Jenssen, H.P.; Linz, A.; Leavitt, R.P.; Morrison, C.A.; Wotman, D.E. Analysis of the optical spectrum of Tm ${ }^{3+}$ in $\mathrm{LiYF}_{4}$. Phys. Rev. B 1975, 11, 92-101. [CrossRef]

14. Meftah, A.; Wyart, J.-F.; Champion, N.; Tchang-Brillet, W.-Ü.L. Observation and interpretation of the $\mathrm{Tm}^{3+}$ free ion spectrum. Eur. Phys. J. D 2007, 44, 35-45. [CrossRef]

15. Kozlov, A.; Dzuba, V.A.; Flambaum, V.V. Optical atomic clocks with suppressed blackbody-radiation shift. Phys. Rev. A 2014, 90, 042505. [CrossRef]

16. Derevianko, A.; Dzuba, V.A.; Flambaum, V.V. Highly charged ions as a basis of optical atomic clockwork of exceptional accuracy. Phys. Rev. Lett. 2012, 109, 180801. [CrossRef] [PubMed]

17. Biémont, E.; Clar, M.; Enzonga Yoca, S.; Fivet, V.; Quinet, P.; Träbert, E.; Garnir, H.-P. Atomic structure calculations and beam-foil observations of La IV. Can. J. Phys. 2009, 87, 1275-1282. [CrossRef]

18. Zhang, Z.G.; Svanberg, S.; Quinet, P.; Palmeri, P.; Biémont, E. Time-resolved laser spectroscopy of multiply ionized atoms-Natural radiative lifetimes in Ce IV. Phys. Rev. Lett. 2001, 87, 273001. [CrossRef] [PubMed]

19. Enzonga Yoca, S.; Quinet, P. Decay rates for radiative transitions in the Pr IV spectrum. J. Phys. B At. Mol. Opt. Phys. 2013, 46, 145003. [CrossRef]

20. Enzonga Yoca, S.; Quinet, P. Relativistic Hartree-Fock calculations of transition rates for allowed and forbidden lines in Nd IV. J. Phys. B At. Mol. Opt. Phys. 2014, 47, 035002. [CrossRef]

21. Wyart, J.-F.; Tchang-Brillet, W-Ü.L.; Spector, N.; Palmeri, P.; Quinet, P.; Biémont, E. Extended analysis of the spectrum of triply-ionized ytterbium (Yb IV) and transition probabilities. Phys. Scr. 2001, 63, 113. [CrossRef]

22. Cowan, R.D. The Theory of Atomic Structure and Spectra; University of California Press: Berkeley, CA, USA, 1981.

23. Dodson, C.M.; Zia, R. Magnetic dipole and electric quadrupole transitions in the trivalent lanthanide series: Calculated emission rates and oscillator strengths. Phys. Rev. A 2012, 86, 125102. [CrossRef]

24. Biémont, E.; Garnir, H.-P.; Bastin, T.; Palmeri, P.; Quinet, P.; Li, Z.S.; Zhang, Z.G.; Lokhnygin, V.; Svanberg, S. Radiative lifetime measurements and transition probabilities of astrophysical interest in Er III. Mon. Not. R. Astron. Soc. 2001, 321, 481-486. [CrossRef]

25. Quinet, P.; Palmeri, P.; Biémont, E.; McCurdy, M.M.; Rieger, G.; Pinnington, E.H.; Wickliffe, M.E.; Lawler, J.E. Experimental and theoretical lifetimes, branching fractions and oscillator strengths in Lu II. Mon. Not. R. Astron. Soc. 1999, 307, 934-940. [CrossRef]

26. Quinet, P.; Palmeri, P.; Biémont, E.; Li, Z.S.; Zhang, Z.G.; Svanberg, S. Radiative lifetime measurements and transition probability calculations in lanthanide ions. J. Alloys Comp. 2002, 344, 255-259. [CrossRef]

27. Quinet, P. An overview of the recent advances performed in the study of atomic structures and radiative processes in the lowest ionization stages of heavy elements. Can. J. Phys. 2017, in press. [CrossRef]

28. Fraga, S.; Karwowski, J.; Saxena, K.M.S. Handbook of Atomic Data; Elsevier: Amsterdam, The Netherland, 1976.

29. Zhang, Z.G.; Li, Z.S.; Svanberg, S.; Palmeri, P.; Quinet, P.; Biémont, E. Experimental and theoretical lifetimes in Yb III. Eur. Phys. J. D 2001, 15, 301-305. [CrossRef]

30. Li, Z.S.; Zhang, Z.G.; Lokhnygin, V.; Svanberg, S.; Bastin, T.; Biémont, E.; Garnir, H.-P.; Palmeri, P.; Quinet, P. Radiative lifetime measurements in Tm III with time-resolved laser spectroscopy and comparosons with HFR calculations. J. Phys. B At. Mol. Opt. Phys. 2001, 34, 1349-1359. [CrossRef]

(C) 2017 by the authors. Licensee MDPI, Basel, Switzerland. This article is an open access article distributed under the terms and conditions of the Creative Commons Attribution (CC BY) license (http:/ / creativecommons.org/licenses/by/4.0/). 